\title{
Reflection and transmission of visible light by sugi wood: effects of cellular structure and densification
}

\author{
Hiroyuki Sugimoto ${ }^{1,2} \cdot$ Sakiko Kawabuchi ${ }^{3} \cdot$ Masatoshi Sugimori $^{1} \cdot$ Joseph Gril $^{2,4}$
}

Received: 9 February 2018 / Accepted: 29 June 2018 / Published online: 3 August 2018

(c) The Japan Wood Research Society 2018

\begin{abstract}
Transmittance and reflectance of visible light by sugi wood (Cryptomeria japonica) were investigated in the longitudinal $(L)$ and tangential $(T)$ directions. Transmittance was the highest in the $L$ direction and reflectance was the highest in the $T$ direction, suggesting that structural anisotropy influences transmittance and reflectance. Intra-ring variations observed with a microspectrometer indicated that $T$ transmittance was higher for latewood than for earlywood, but there was no such trend in for $L$ transmittance in which the highest levels occurred near the annual ring boundaries, on either the earlywood or latewood side, and the lowest at the transition from earlywood to latewood. Dependence of $L$ transmittance on wavelength also showed variations according to the intra-ring position. The increasing of transmittance of earlywood at wavelengths $<500 \mathrm{~nm}$ with increasing wavelength was observed, but this was not confirmed for latewood because of absorption by lignin. These observations supported a previously published finding, which was based on measurements in the radial direction, that the number of internal cell wall reflections, rather than density, determines wood lightness. Indeed, in the $L$ direction, most of the incident light passes through lumens in earlywood and through cell walls in latewood, while it is subjected to numerous internal reflections at the interface between lumens and cell walls. This was further confirmed by the transmittance of earlywood being greatly decreased by radial compression.
\end{abstract}

Keywords Visible light $\cdot$ Cell structure $\cdot$ Transmittance $\cdot$ Reflection $\cdot$ Compression

\section{Introduction}

Owing to its esthetic characteristics, wood is used for the manufacture of many high-value products [1]. Therefore, the appearance of wood, such as its texture, grain patterns, or color, has been studied. Studies investigating the lightness of the wood surface have also been conducted by many

Hiroyuki Sugimoto

sugimoto.hiroyuki.rw@ehime-u.ac.jp

1 Ehime University Graduate School of Agriculture, Tarumi, Matsuyama 790-8566, Japan

2 LMGC, Univ. Montpellier, CNRS, CC4048, 163 rue Auguste Broussonnet, 34090 Montpellier, France

3 Abashiri Nambu District Forest Office, Forest Agency, Ministry of Agriculture, Forestry and Fisheries, 656-3, Koshimizu, Koshimizu-cho, Shari-gun, Hokkaido 099-3632, Japan

4 CNRS, Université Clermont Auvergne, Sigma Clermont, Institut Pascal, Campus des Cezeaux, 2 av. Blaise Pascal, TSA 60206, CS 60026, 63178 Aubière Cedex, France researchers [2-5], and in general, it is believed that wood with higher density tends to be darker in terms of light absorption. However, this could not always be explained well by the density dependence of wood lightness. Palviainen et al. [6] investigated the influence of density on diffraction using 30 wood species. They showed that samples with lower densities are likely to produce more scattering. However, such samples of different species also have different anatomical features. In a previous study [7], we measured total light transmittance and total reflectance of sugi (Cryptomeria japonica) compressed at various levels. The results showed that in medium- and long-wavelength regions, the relationship between transmission and reflection is strong because the influence of cell wall absorption is small. That is, the effect of reflection on lightness in the long-wavelength region is as comparable to that of absorption by the cell wall.

The Kubelka-Munk (KM) theory based on specific scattering coefficient and specific absorption coefficient is frequently used for paper or powder [8] to understand the spectroscopic characterization. However, KM theory only deals 
with the propagating energy of incident light from the front to the rear, parallel to the direction of propagation in the case of a material that is homogeneous along its thickness; it may thus be difficult to apply to wood given its inhomogeneous structure $[9,10]$. Moreover, this theory supposes that the incident light mainly pass the substance. In wood, the volume ratio of air to cell wall varies greatly depending on the species and tree part, so that light might pass through air in lumen as much as through the cell wall, in a proportion depending on that ratio. Therefore, we need a model for light transmission and reflection considering not only the cell wall material [11] but also cell structure.

In a previous study, focused on the influence of density on the transmittance/reflectance of visible light, the compression and measurement directions were fixed in the radial direction. In this study, to examine the influence of cell structure, the transmittance and reflectance of light were measured in both longitudinal and tangential directions. Light properties in earlywood and latewood were compared using a microspectrometer. Moreover, to examine the effect of compression treatment, measurements in a direction different from that of the compression were performed, along with observation using microtomography.

\section{Materials and methods}

\section{Sample preparation}

Wood blocks were taken from sugi (Cryptomeria japonica) sapwood. The sample dimensions were $64 \mathrm{~mm}$ in the tangential direction $(T) \times 47 \mathrm{~mm}$ in the radial direction $(R) \times 142 \mathrm{~mm}$ in the longitudinal direction $(L)$. To measure density, one portion of $10 \mathrm{~mm}$ in the $L$ direction was cut from each of these blocks, and the remaining parts were used for visible light spectroscopy measurements. Density was measured by the double-weight method. Prior to visible light spectroscopy, some specimens were subjected to compression treatment: after being steamed at $100{ }^{\circ} \mathrm{C}$ for $2 \mathrm{~h}$, they were compressed in the $\mathrm{R}$ direction using a press machine (Denki Netsuban; SHINTO Metal Industries Corp.) equipped with a flat heating metallic die polished to a mirror finish. Neglecting the deformation in the T direction resulting from the compression, we defined a compression ratio (CR) varying from zero for an uncompressed state to unity for a fully compressed state as follows:

$\mathrm{CR}=\left(d_{0}-d\right) /\left(d_{0}-d_{\mathrm{L}}\right) \times 100$,

where $d_{0}, d$, and $d_{\mathrm{L}}$ are the original, compressed, and limit heights of the specimen, respectively. The limit height is calculated by dry weight divided by cell wall density of 1.5 and by base area.
After being compressed to the predefined thickness, each sample was left for $24 \mathrm{~h}$ and then left to stand in a desiccator. After 1 week, the dimensions and weight were measured.

For visible light spectroscopy measurement, compressed samples were cut by a rim saw into 10 specimens of various thicknesses of $0.3-1.5 \mathrm{~mm}$ in the $L$ direction; the remaining parts were also cut into 10 specimens of various thicknesses of $0.3-1.5 \mathrm{~mm}$ in the $T$ direction.

\section{Visible light spectroscopy measurement}

The total light reflectance and total light transmittance in the wavelength range from 350 to $800 \mathrm{~nm}$ were measured at $20{ }^{\circ} \mathrm{C}$ using a UV-Vis spectrophotometer (V-670; JASCO Co. Ltd.) equipped with an integrating sphere.

Irradiation light energy within a bandwidth of $5 \mathrm{~nm}$ of this device in the visible light region was about $1 \mu \mathrm{W}$ and the spot diameter was about $5 \mathrm{~mm}$. The samples were cut into $15-\mathrm{mm}$ squares to fit the cell. A barium sulfate white plate was used as the reference sample.

\section{Image analysis by X-ray CT}

The images were observed using an X-ray CT scanner (Skyscan 1272 Bruker MicroCT). After spectroscopy measurement, the samples were cut into sections of $5 \mathrm{~mm}$ in the $T$ direction. The observation conditions were $50 \mathrm{kV}, 200 \mu \mathrm{A}$, $0.2^{\circ}$ rotation, and $1.2 \mu \mathrm{m}$ pixel size at a temperature of $20^{\circ} \mathrm{C}$ and relative humidity of $60 \%$. The obtained data were reconstructed using NRecon software. CTVO software was used for $3 \mathrm{D}$ visualization.

\section{Transmittance measurement using UV-Visible/NIR micro-spectrometers}

The transmittance at the micro-scale in the visible light region was measured by UV-Visible/NIR micro-spectrometers (MSV-5200; JASCO Co. Ltd.). The measurement diameter was $100 \mu \mathrm{m}$. Four points in earlywood and four points in latewood were measured for each specimen. Further, for the uncompressed $L$ sample, 20 points were measured along two annual rings.

\section{Results and discussion}

\section{Difference of transmittance and reflectance between $L$ and $T$ directions}

Figure 1 shows the influence of sample thickness on total light transmittance $(\tau)$ and reflectance $(\rho)$ of sugi wood in the $L$ and $T$ directions at the wavelength of $750 \mathrm{~nm}$. Although the reflectance of the $L$ sample did not exhibit a clear trend, 

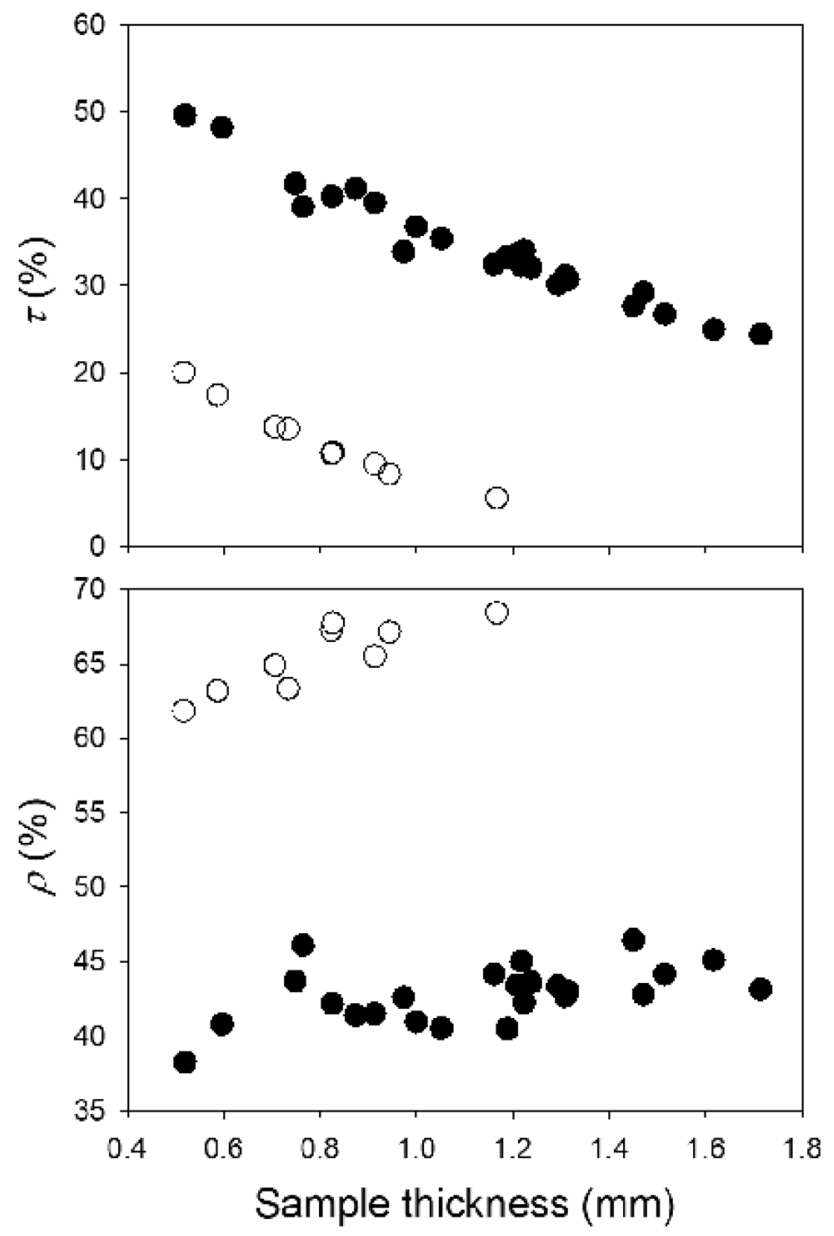

Fig. 1 Influence of sample thickness on total light transmittance $(\tau)$ and reflectance $(\rho)$ of sugi wood in the longitudinal direction (filled circles) and tangential direction (open filled circles)

$\tau$ decreased and $\rho$ increased when the thickness increased, as reported previously. Moreover, $\tau$ of the $L$ sample was larger than that of the $T$ sample. $\rho$ showed the opposite trend. These results suggest that $\tau$ and $\rho$ properties are affected by the anisotropy of wood cell structure.

The annual ring structure is the most clearly recognized wood feature involving a contrast of brightness. Therefore, we started by comparing the difference of $\tau$ between earlyand latewood, as observed using micro-spectrometers. $\tau$ of all samples was small at short wavelengths up to $400 \mathrm{~nm}$. Moreover, $\tau$ of latewood in all $T$ samples was larger than that of earlywood. Typical results of the $T$ sample with a thickness of $5 \mathrm{~mm}$ are shown in Fig. 2. In previous paper [7], we predicted the $\tau$ by the expression of the number of the cell wall/lumen interfaces. In $T$ samples, the incident light will pass through a series of cell wall/lumen reflection interfaces, especially in earlywood, as can be predicted by the expression. However, in latewood, where cells have a thick cell wall and a small lumen diameter, in addition to

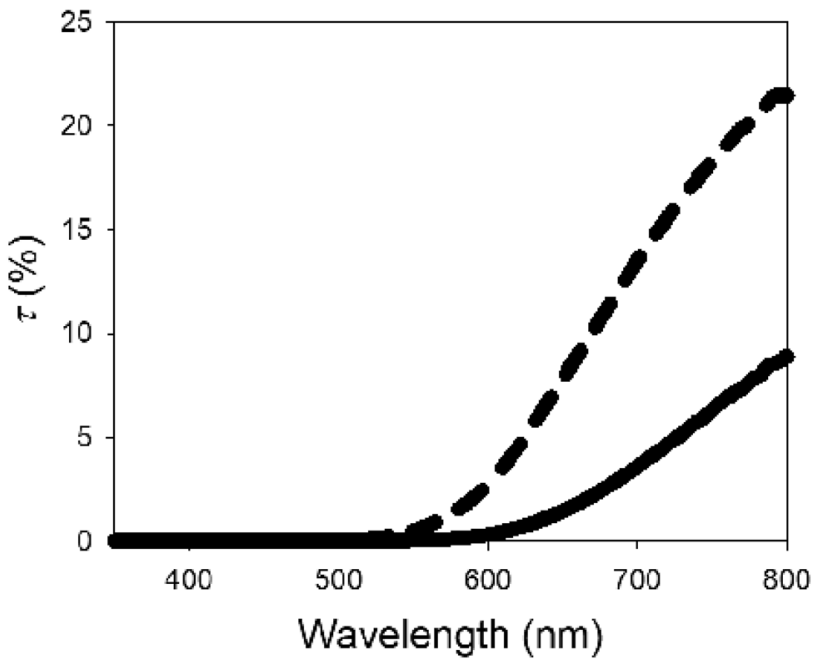

Fig. 2 Comparison of wavelength dependence of total light transmittance $(\tau)$ between earlywood (solid line) and latewood (dashed line) in the $T$ direction for a thickness of $0.52 \mathrm{~mm}$

being well aligned in both $R$ and $T$ directions, the incident light often passes through the cell wall without encountering the interface. This explains why $\tau$ of latewood is higher than that of earlywood. On the other hand, this trend was not observed in the $L$ sample. This suggests the possibility that other mechanisms are involved in the $L$ direction.

Figure 3 compares the cell structure image obtained by micro-CT scanning and the corresponding $\tau$ measured by a microspectrometer, at the wavelength of $750 \mathrm{~nm}$. The transmittance greatly varied depending on the measurement location. From the pith (right) side, $\tau$ of the earlywood (EW) was over $35 \%$; it dropped to $<35 \%$ in the transition zone (TW), increased up to $45 \%$ in the latewood (LW) toward the boundary of the annual ring $(\mathrm{LWb})$, and then recovered to the same level of $>35 \%$ in the next EW zone. $\tau$ most likely depends on the location even within the same LW.

Figure 4 shows the dependence of $\tau$ in the $L$ direction on wavelength in sugi wood at various locations and its logarithmic curves. For short wavelengths up to $500 \mathrm{~nm}$, there was an increase of $\tau$ in EW with increasing wavelength in the curves, but this was not confirmed for TW and LW. Therefore, the light path differs between EW and LW; higher values of EW transmittance are possibly because of lower light absorption. It is assumed that absorbance at wavelengths up to $600 \mathrm{~nm}$ is because of the existence of lignin [12]. Lower $\tau$ of TW and LW than that of EW suggests that the light in TW and LW passes through the cell wall more than that in EW. In contrast, for long wavelengths, the $\tau$ of LW is greater than that of $\mathrm{EW}$. In TW, at the transition from EW to $\mathrm{LW}, \tau$ is the lowest. Tsuchikawa and Tsutsumi showed that, for short wavelengths in the near-infrared region, in other words, for long-wavelength visible light, scattering occurs 
Fig. 3 Cell structure image by $\mathrm{X}$-ray CT of $L$ sample with a thickness of $0.60 \mathrm{~mm}$ and total light transmittance $(\tau)$ at given positions of the same sample

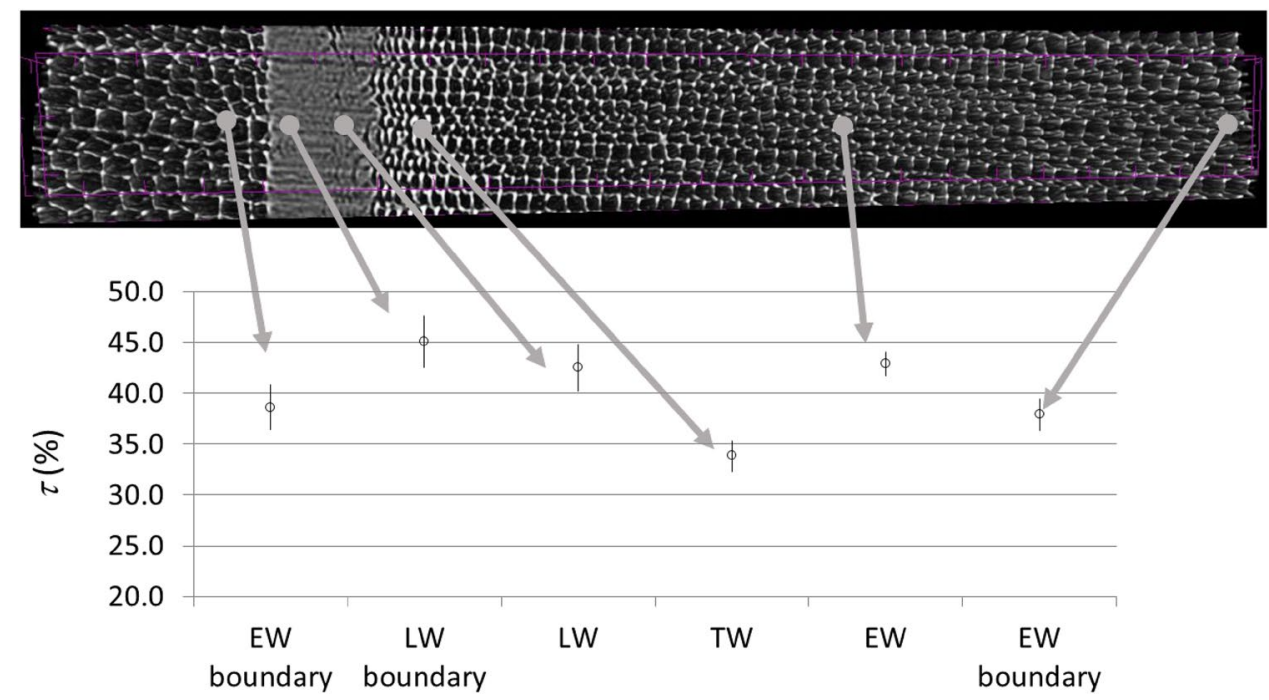

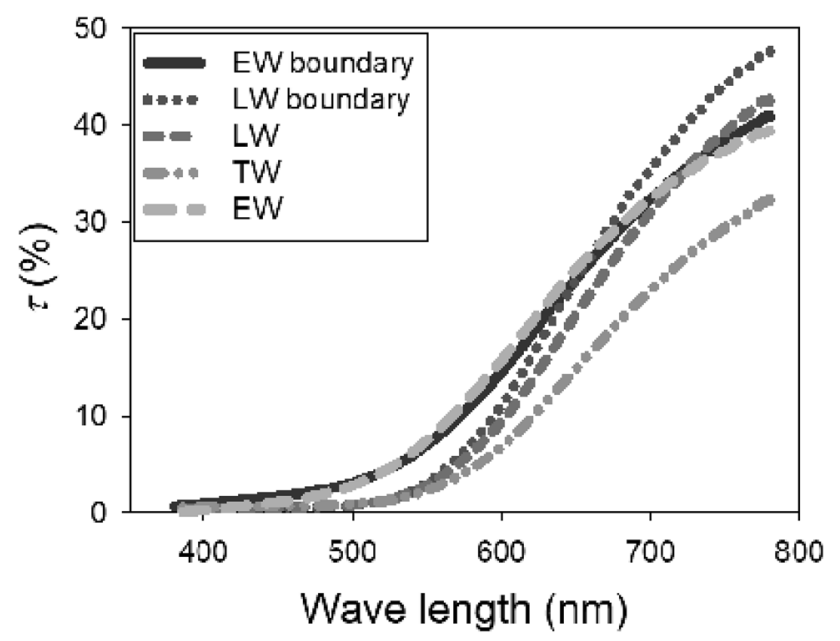

Fig. 4 Dependence of total light transmittance $(\tau)$ in the $L$ direction on wavelength at the positions shown in Fig. 3

in a direction perpendicular to that of incident light [13]. Therefore, the light path in this part is longer than that in other parts due to the occurrence of substantial scattering.

For long wavelengths, the $\tau$ of LW is greater than that of EW because the light passes through the cell wall with less numerous interfacial reflections. Furthermore, light passing through the cell wall may hardly diffuse because of total reflection at the cell wall/lumen interface.

This can be qualitatively confirmed by image evaluation. Figure 5 shows an image with the light from the photographing side (left) and from the back of the sample (right). The former corresponds to reflected light and the latter to transmitted light. In the left image, LW is darker than EW, whereas in the right image it is lighter and more scattered; in particular, the light is most visible close to annual ring boundaries. Moreover, the darkest part is TW. These trends agree well with the microspectrometer results.

If the above-mentioned assumption that TW is the darkest owing to numerous interfaces is correct, the increase in density owing to compression should not necessarily lead to an increase in $\tau$ because compression treatment in a direction different from that of the light path does not decrease the number of interfaces between cell wall and lumen.

\section{Change in transmittance by compression}

To quantify the effect of compression, the relationship between $\tau$ and $\rho$ and the thickness in the $L$ and $T$ directions of radially compressed samples with various compression ratios is shown in Fig. 6. Both $\tau$ and $\rho$ of compressed samples were lower than those of uncompressed wood. These results are contrary to those in a previous paper [7]. The light in the compressed part may have been scattered toward the outside of the integrating sphere. For a given thickness, the interfaces of refraction and the consequent increasing of light pass are increased by compression treatment. To investigate the relationship between the change of cell structure resulting from the compression and the light properties, images of cell structure obtained by X-ray CT scanning are shown in Fig. 7 and $\tau$ in $T$ direction of earlywood of compressed samples measured by microspectrometer is shown in Fig. 8. Calculated values are shown for comparison, based on the following model [7]. We considered the case of a regular arrangement of cells with a square cross section of width $30 \mu \mathrm{m}$ and thin cell wall (which is the case of sugi wood). We neglect the direct transmission of light through the thin cell walls disposed parallel to the light direction, and only consider the reflection occurring at the interface between lumens 
Fig. 5 Comparison of an image of $L$ sample with a thickness of $0.76 \mathrm{~mm}$ with the light from the photographing side (left) and from the back of the sample (right)

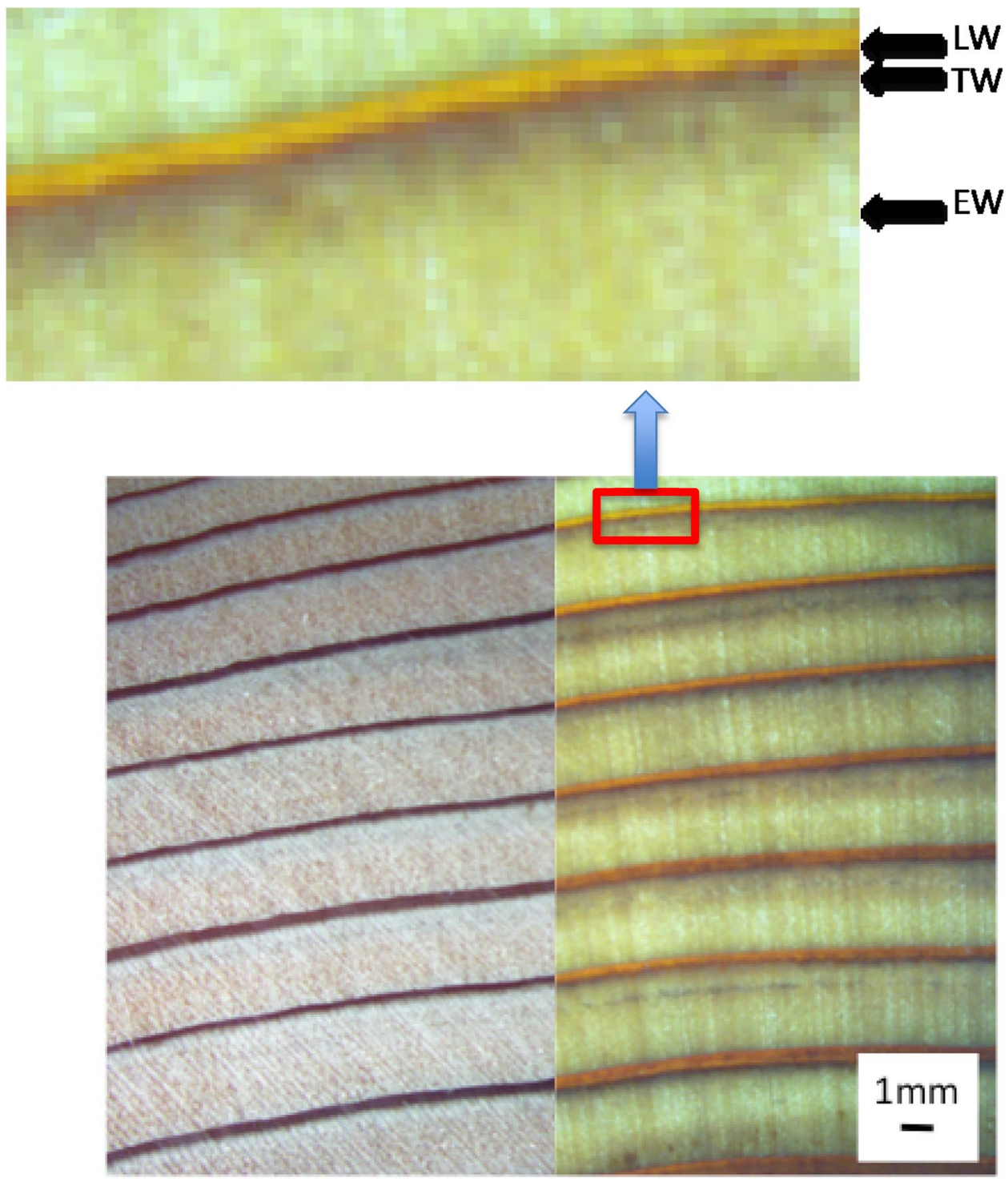

and transverse cell walls. The sample thickness being $D$ $(\mu \mathrm{m})$, the number of such reflections equals $D / 15$ and the total transmittance $(\tau)$ can be described by

$\tau=\tau_{\mathrm{i}}^{\frac{D}{15}}$,

where the transmittance of each interface $\left(\tau_{\mathrm{i}}\right)$ is given by

$\tau_{\mathrm{i}}=\frac{4 n_{0} n_{1}}{\left(n_{0}+n_{1}\right)^{2}}$

assuming a refractive index for the cell wall $\left(n_{1}\right)$ of 1.6 [14] and for air $\left(n_{0}\right)$ of 1 .

Comparing the compressed and uncompressed samples in terms of end grain, the cell wall of earlywood collapsed in an intricate way.
This change of cell structure may have increased the number of diffused reflections in the earlywood per measurement area because of more numerous interfaces resulting from the intricate cell wall even so steaming treatment affects $\tau$ [15]. The lower $\rho$ values of compressed samples than that of uncompressed samples are in good agreement with practical appearance of lightness of earlywood (Fig. 9).

\section{Conclusion}

To investigate the effects of cellular structure and densification on total light transmission and reflection in the visible light region, the anisotropy of total light transmittance $(\tau)$ and reflectance $(\rho)$, as well as the micro-local $\tau$ of 

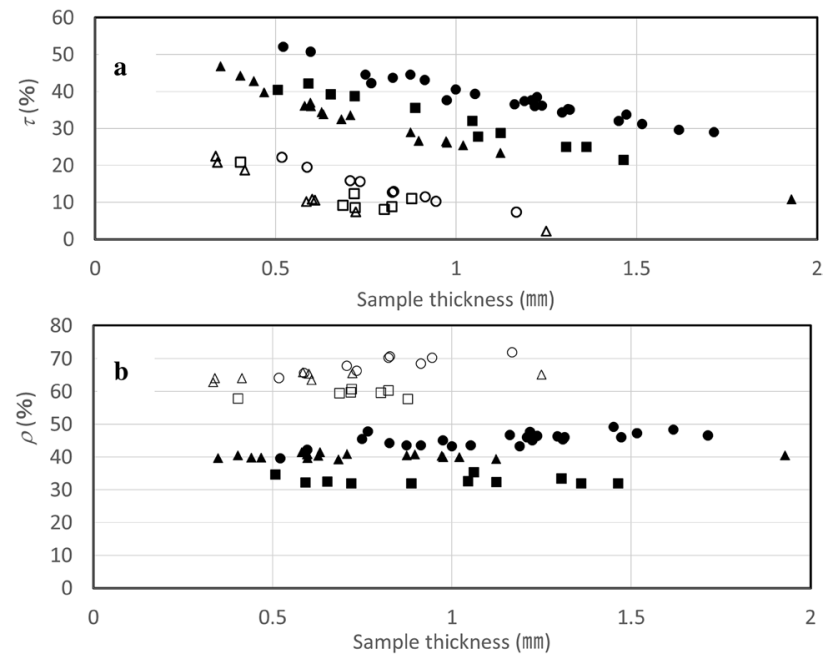

Fig. 6 Relationship between a total light transmittance $(\tau)$ or $\mathbf{b}$ reflectance $(\rho)$ and the thickness in $L$ (close) and $T$ (open) directions of compressed samples with various compression ratios (circle: uncompressed, triangle: $81 \%$, square: $87 \%$ )

uncompressed and compressed wood, was measured. Based on the obtained results, the following conclusions can be drawn:

- $\tau$ in the $L$ direction is higher than that in the $T$ direction. In all $T$ direction samples, $\tau$ of latewood was higher than that of earlywood. Conversely, in the $L$ direction, a similar trend was not observed.

- Although $\tau$ of late- and earlywood near the annual ring boundaries was high, in the transition from early- to latewood, it was the lowest. These facts suggest that in the $L$ direction, most of the incident light passed through lumens in earlywood and through cell walls in latewood, while in the transition part, diffusion due to light passing lumen/wall interfaces occurs more often, resulting in a lower $\tau$.

- For short wavelengths up to $500 \mathrm{~nm}$, there is an increase of $\tau$ of EW with increasing wavelength in the curves, but this was not confirmed for TW and LW. Absorbance up to $600 \mathrm{~nm}$ caused by lignin, and the thinner cell walls of EW could explain that observation. At longer wavelengths, $\tau$ of latewood is greater than that of earlywood. These results support the previous deduction that differences of $\tau$ among the parts in rings are because of the different light paths.

- The transmittance of the earlywood was greatly reduced by compression treatment, probably because of numerous internal reflections at the cellular level. The lower $\rho$ of compressed samples than that of uncompressed samples are in good agreement with practical appearance of lightness of earlywood.

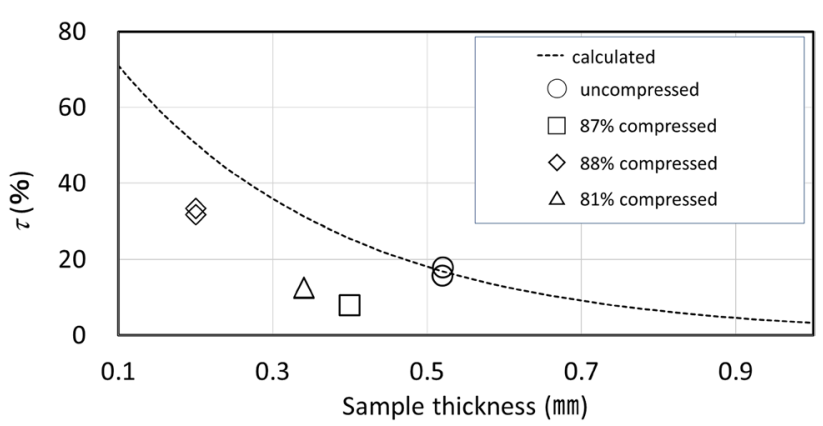

Fig. 8 The relationship between total light transmittance $(\tau)$ in $T$ direction of earlywood of compressed samples measured by microspectrometer with various compression ratios and sample thicknesses; dashed line, calculation based on model/equation
Fig. 7 Image of the cell structure of compressed wood using $\mathrm{X}$-ray CT scanning

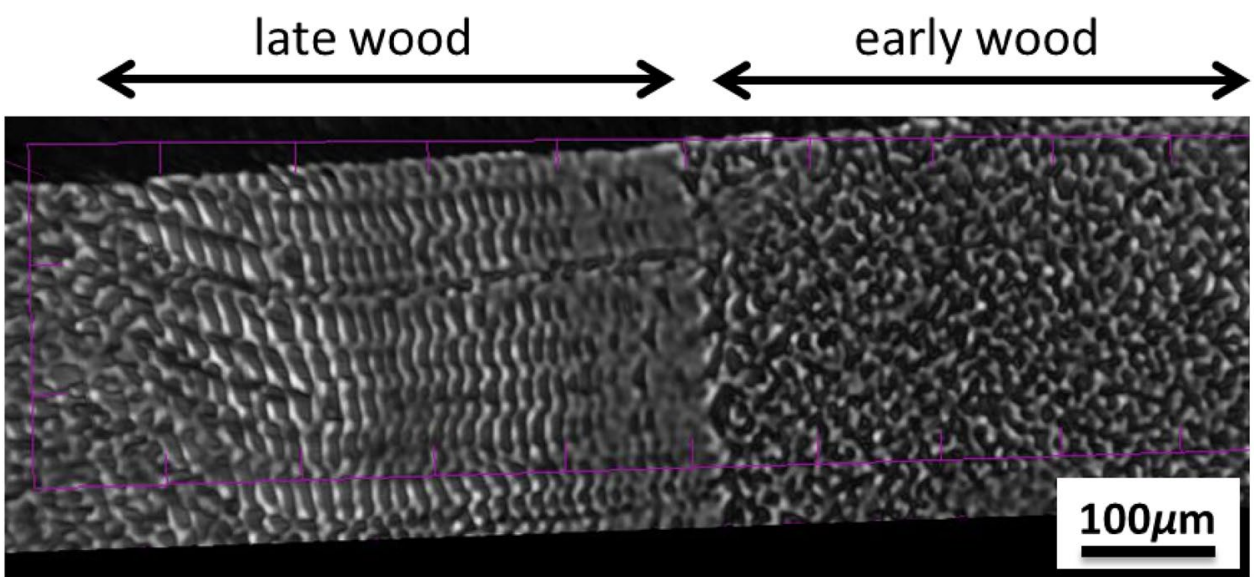


Fig. 9 Images of the $L$ samples (upper) and the $T$ samples (lower) with compressed treatment (right) and uncompressed (left). Measured light properties at framed red squares
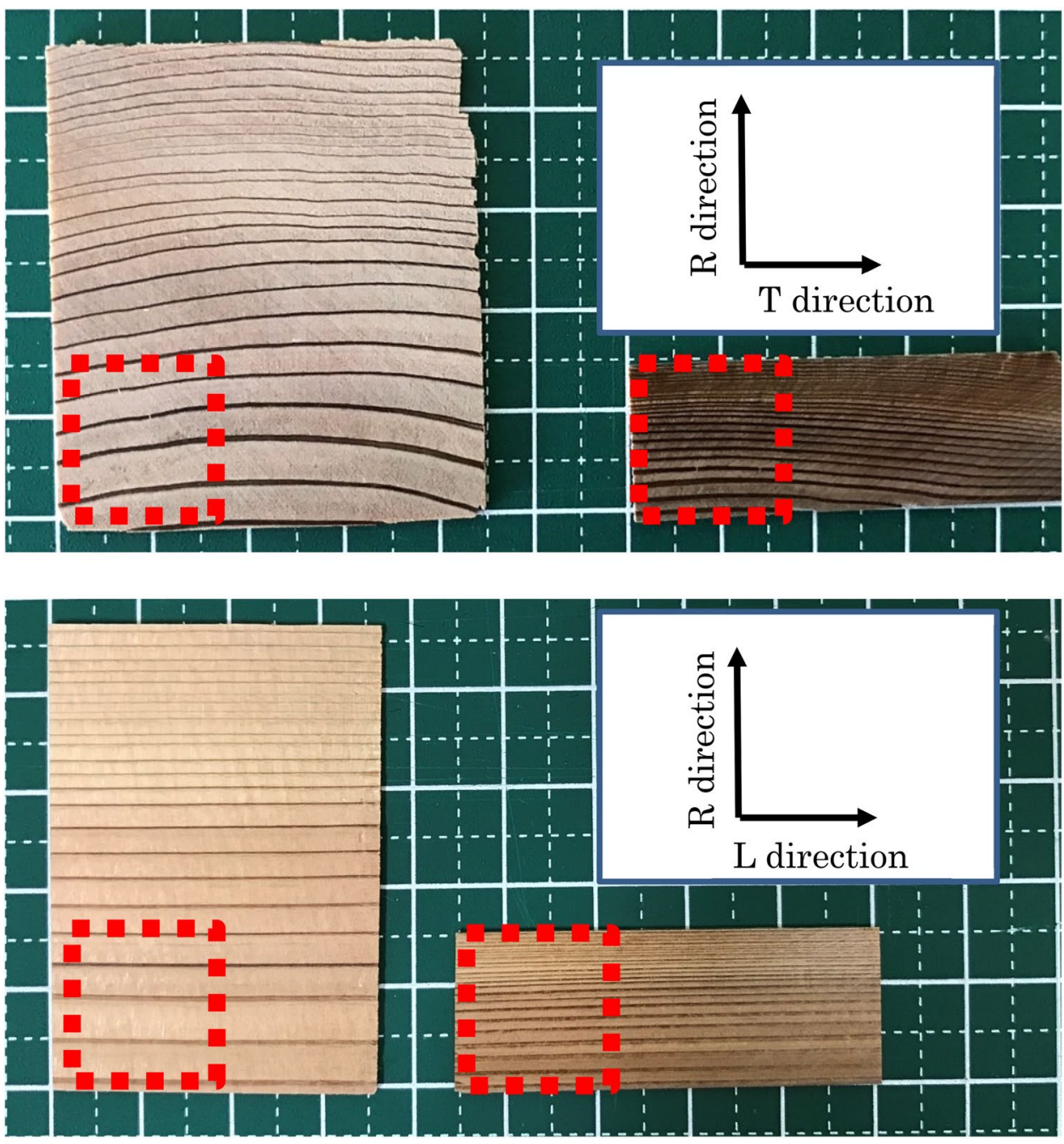

\section{References}

1. NTT docomo HP (in Japanese). https://www.nttdocomo.co.jp/ info/news_release/2011/03/09_00.html. Accessed 20 July 2018

2. Kitamura Y (1987) Measured colour values of important domestic and imported wood (in Japanese). Bull For Forest Prod Res Inst 347:203-239

3. Kataoka Y, Kiguchi M, Williams RS, Evans PD (2007) Violet light causes photo degradation of wood beyond the zone affected by ultraviolet radiation. Holzforschung 61:23-27

4. Mitsui K, Takada H, Sugiyama M, Hasegawa R (2005) Changes in the properties of light-irradiated wood with heat treatment. Part 1. Effect of treatment conditions on the change in color. Holzforschung 55:601-605

5. Ban M, Inagaki T, Ma T, Tsuchikawa S (2018) Effect of cellular structure on the optical properties of wood. J Near Infrared Spectrose 26:53-60

6. Palviainen J, Silvennoinen R (2001) Inspection of wood density by spectrophotometry and a diffractive optical element based sensor. Meas Sci Technol 12:345-352

7. Sugimoto H, Rikitake T, Sugimori M (2018) Optical reflection and transmission of sugi wood in visible light (in Japanese). Mokuzai Gakkaishi 64:66-71

8. Scallan AM, Borch J (1972) An interpretation of paper reflectance based upon morphology I. Initial considerations. Tappi 55:583-588
9. Tolvaj L, Mitsui K, Varga D (2011) Validity limits of KubelkaMunk theory for DRIFT spectra of photodegraded solid wood. Wood Sci Techol 45:135-146

10. Tsuchikawa S, Tsutsumi S (1996) Application of near infrared spectrophotometry to wood III. Behavior of reflected and transmitted light for parallel beam of incident light (in Japanese). Mokuzai Gakkaishi 42:733-742

11. Saarinen K, Muinonen K (2001) Light scattering by wood fibers. Appl Opt 40:5064-5077

12. Mononen K, Jääskeläinen AS, Alvila L, Pakkanen TT, Vuorinen T (2005) Chemical changes in silver birch (Betula pendula Roth) wood caused by hydrogen peroxide bleaching and monitored by color measurement (CIELab) and UV-Vis, FTIR and UVRR spectroscopy. Holzforschung 59:381-388

13. Tsuchikawa S, Tsutsumi S (1996) Application of near infrared spectrophotometry of wood II. Behavior of irradiated light in an aggregate of tracheids and directional characteristics of diffuse reflected and transmitted light (in Japanese). Mokuzai Gakkaishi 42:343-353

14. Fink S (1992) Transparent wood-a new approach in the functional study of wood structure. Holzforschung 46(5):403-408

15. Bourgois P, Janin G, Guyonnet R (1991) The color measurement: a fast method to study and to optimize the chemical transformations undergone in the thermically treated wood (in French). Holzforschung 45:377 